\title{
van der Waals corrected density functional calculations of the adsorption of benzene on the $\mathrm{Cu}(111)$ surface
}

\author{
Damien J. Carter, ${ }^{1}$ and Andrew L. Rohl ${ }^{1}$ \\ Correspondence to: Damien Carter (E-mail: d.carter@curtin.edu.au)
}

\begin{abstract}
${ }^{1}$ Damien J. Carter and Andrew L. Rohl
Nanochemistry Research Institute \& Department of Chemistry, Curtin University, GPO Box U1987, Perth WA,
\end{abstract} Australia, 6845

\section{ABSTRACT}

We investigate the performance of several vdW functionals at calculating the interactions between benzene and the copper (111) surface, using the local orbital approach in the SIESTA code. We demonstrate the importance of using surface optimised basis sets to calculate properties of pure surfaces, including surface energies and the work function. We quantify the errors created by using (3x3) supercells to study adsorbate interactions by using much larger supercells, and show nonnegligible errors in the binding energies and separation distances. We examine the 8 high-symmetry orientations of benzene on the $\mathrm{Cu}(111)$ surface, reporting the binding energies, separation distance and change in work function. The optimised vdW-DF(optB88-vdW) functional provides superior results to the vdW-DF(revPBE) and vdW-DF2(rPW86) functionals, and closely matches the experimental and experimentally deduced values. This work demonstrates that local orbital methods using appropriate basis sets combined with a vdW functional can model adsorption between metal surfaces and organic molecules.

\section{Introduction}

Density functional theory (DFT) is one of the most accurate methods for determining the stability and properties of structures; however until recently these relatively fast calculations were not commonly being used to examine soft matter or biomolecular or molecular crystals. The lack of a description of van der Waals ( $v d W)$, or dispersion, forces was the overriding factor, because these forces make a large and important contribution in these types of systems.

The development of functionals that include $\mathrm{vdW}$ forces is an active research field, and there are now well-established methods for including these types of interactions in DFT (see a recent review by Grimme ${ }^{1}$ ). One of the simplest and most popular methods to include vdW forces has been with an empirical correction using interatomic potentials of the form $C_{6} R^{-6}$, where $C_{6}$ is the dispersion coefficient, and $R$ is the pairwise atom distance. ${ }^{2-6}$ Early implementations had coefficients that depended on the fitting environment, which limited the transferability and accuracy. A number of methods were developed to overcome this issue, including the TkatchenkoScheffler (TS) approach ${ }^{7}$ and the local response dispersion method, ${ }^{8}$ and Grimme et al. later refined their method for computing coefficients to interpolate between dispersion coefficients for atoms in different chemical environments, referred to as DFT-D3. ${ }^{9}$ Related to these approaches is the exchange-hole dipole (XDM) method of Becke and Johnson. ${ }^{10-13}$ Other common approaches to incorporate vdW forces in DFT include adapting semi-local and hybrid functionals to include medium and long-rang correlations effects, ${ }^{14-17}$ or by incorporating non-local correlation effects using wavefunction 
theory. ${ }^{18,19}$ The vdW-DF approaches use an explicit nonlocal correlation term to describe the dispersion, and these approaches have been combined with a variety of exchange functionals. ${ }^{20-24}$

The adsorption of organic molecules, in particular aromatics, on metal surfaces is a matter of intensive research as many material properties can be extensively modified by these molecules. One of the important uses of noble metals is for catalysts in hydrogenation and cracking reactions. In the context of molecular electronics, monolayers of molecules at the electrode can be used to tune the work function. ${ }^{25}$ Molecular adsorption can also lead to enhanced chemical sensing ${ }^{26}$ or corrosion resistance. ${ }^{27}$

$A b$ initio calculations studying interactions of organic molecules with surfaces of copper have been used to investigate a range of adsorbates including benzene, ${ }^{28-40}$ corannulene, ${ }^{41}$ fluorobenzenes, $^{40}$ graphene, $^{42}$ thiophene, ${ }^{36}$ pyridine, ${ }^{35,36}$ and 3,4,9,10-perylenetetracarboxylicaciddianhydride. ${ }^{43}$ Jenkins ${ }^{44}$ also recently published a review of first principles calculations of the interactions of aromatic molecules on metal surfaces, including copper. The majority of these studies examine interactions with $\mathrm{Cu}$ (111) surfaces, although some studies consider the $(100)^{34}$ and $(110)^{40}$ surfaces. The large number of different types of calculations of benzene on the $\mathrm{Cu}$ (111) surface is in part due to the wealth of available experimental data, including information from high-resolution electron energy loss spectroscopy, ${ }^{45}$ photoelectron spectroscopy, ${ }^{38}$ low-energy electron diffraction, ${ }^{46}$ near-edge $x$ ray absorption fine structure, ${ }^{45}$ high-resolution angle-resolved photoemission ${ }^{47}$ and scanning tunneling microscopy. ${ }^{34}$

DFT calculations of benzene on the $\mathrm{Cu}$ (111) surface have used a wide range of functionals, with varying degrees of success, and include methods and functionals that don't account for vdW forces, such as $\mathrm{PW} 1^{30}$ and $\mathrm{PBE},{ }^{37}$ and those that do, which include $v d W$ -
DF(revPBE), ${ }^{29,32,37,39}$ vdw-DF2(rPW86), ${ }^{32,39}$ and PBE-XDM. ${ }^{33}$ Wavefunction methods, such as MP2, ${ }^{28,31,38}$, have also been used. Despite the number of available studies there are a number of open questions that remain or improvements that could be made. Some studies correct the energetics using vdW-DF or other functionals/methods, but calculate geometries optimized using functionals that don't include vdW forces, such as the PBE functional, ${ }^{29,37}$ which leads to errors in the separation distance, and subsequently affects the binding energy and change in work function. Almost all studies above use a $(3 \times 3)$ supercell to examine the interaction of benzene on the $\mathrm{Cu}(111)$ surface, with some authors ${ }^{29}$ suggesting that there is an error due to adsorbate-adsorbate interactions (i.e. between periodic images of the benzene molecules), and others suggesting that this effect is negligible, ${ }^{32}$ with no quantitative analysis of this possible error.

In this study we investigate the performance of several vdW-DF functionals in studying the interactions of benzene on the copper (111) surface with the local orbital approach of the SIESTA code. ${ }^{48}$ We have recently published several important precursor studies using vdWDF calculations, ${ }^{49,50}$ where we optimized the choice of basis set and functional to get the best result for both gas phase and condensed phases. Here we first investigate the effects of using surface optimized basis sets to calculate electronic properties of pure $\mathrm{Cu}$ (111) surfaces. Garcia-Gil et al. ${ }^{51}$ recently demonstrated the importance of using surface optimized basis sets for metal surfaces using the local orbital approach of SIESTA with the PBE functional. Next changes in the surface energy and work function are examined to assess the performance of vdW-DF functionals using these surface optimized basis sets. Then the effects of supercell size on the binding energy and separation distance of benzene are quantified by examining $(3 \times 3),(4 \times 4),(5 \times 5)$ and $(7 \times 7)$ supercells. Finally the interactions of benzene on the Cu (111) surface are examined using the 8 high symmetry configurations of benzene, and 
the binding energy, separation distance and change in work function for each functional calculated.

\section{Computational Details}

DFT calculations are performed with the SIESTA $^{48}$ code using the vdW-DF(revPBE), ${ }^{21}$ vdW-DF(optB88-vdW), ${ }^{52}$ vdW-DF2(rPW86), ${ }^{22}$ and revPBE ${ }^{53}$ exchange-correlation functionals. Effective potentials due to the nucleus and core electrons are treated using norm-conserving Troullier and Martins ${ }^{54}$ pseudopotentials. Hartree and exchange correlation energies are evaluated on a uniform real-space grid of points with a defined maximum kinetic energy of 300 Ry. Copper atoms are described using a triplezeta plus polarisation (TZP) basis set adapted from Garcia-Gil et al. ${ }^{51}$ Carbon and hydrogen atoms are described with a TZP basis set using a cutoff of 7 Bohr for the $s$ orbitals, with a softconfinement potential of $20 \mathrm{Ry}$, soft inner radius of 0.90 , and split norm values of 0.15 , 0.15 and 0.5 for $\mathrm{Cu}, \mathrm{C}$ and $\mathrm{H}$ atoms, respectively (see Junquera et al. $^{55}$ for more detail).

For each exchange-correlation functional we optimised bulk fcc copper using a $12 \times 12 \times 12$ k-point grid. We then generated a (1x1) surface slab (vacuum region of approximately $30 \AA$ ) of the (111) surface using a 7-layer slab model. This is consistent with other calculations of benzene on the $\mathrm{Cu}(111)$ surface that use either a 7-layer ${ }^{30,33}$ or a 6-layer ${ }^{29,32,37}$ model. To verify the convergence, we examined the surface energy and work function for unrelaxed surfaces containing 7,13 and 19 atomic layers, and found the differences from the values with 7-atomic layers was less than approximately 1 percent.

In addition to using the TZP (bulk) basis set for calculations of the $\mathrm{Cu}$ (111) surface, we also created 3 surface optimised basis sets: the first increased the $4 \mathrm{~s}$ orbital cutoff radius to $12 \mathrm{Bohr}$ (referred to as "optimized"), while the second and third basis sets used floating (or ghost) orbitals located above the surface at positions where the next atomic layer (referred to as "1- float") or the next two atomic layers (referred to as "2-float") would have been in the fcc stacking sequence. Garcia-Gil et al. ${ }^{51}$ previously demonstrated the importance of surface optimized basis sets using the PBE functional. We examine changes in the surface energy and work function to assess the performance of vdW functionals using surface optimized basis sets. For these calculations of the clean $\mathrm{Cu}$ (111)

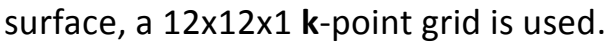

To investigate the interactions of benzene on the $\mathrm{Cu}$ (111) surface with the vdW-DF(revPBE), vdW-DF(optB88-vdW), vdW-DF2(rPW86) and revPBE functionals, we generated a $(5 \times 5)$ surface supercell containing 25 copper atoms per layer. The 5 inner layers of the surface slab are kept fixed, while the outermost layer on each side of the slab (and any ghost orbitals, if present) and the benzene molecules are fully optimized with each particular functional. We examined the eight high-symmetry binding configurations on the surface, denoted BR-I, FCC-I, HCP-I, TOP-I, BR- $R$, FCC- $R$, HCP- $R$ and TOP- $R$ (where $I$ refers to "in-phase", and $R$ refers to a $30^{\circ}$ rotation of the benzene, as described by Berland et al. ${ }^{29}$ ) All binding energy calculations used the 1-float surface optimized

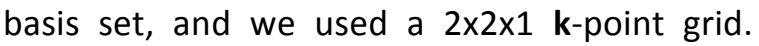
Binding energy calculations were performed on both sides of the surface slab to avoid the need for dipole corrections. Binding energies $\left(E_{B}\right)$ are calculated using $E_{B}=\left(E_{\text {total }}-\left(2 x E_{\text {benz }}+E_{\text {surf }}\right)\right) / 2$, where $E_{\text {total }}$ is the energy of the surface slab with benzene molecules on each surface, $E_{\text {benz }}$ is the energy of an isolated benzene molecule and $E_{\text {surf }}$ is the energy of the clean surface slab. To account for basis set superposition errors (BSSE) in molecular systems we used the standard counterpoise correction (CP) method. ${ }^{56}$ To account for BSSE in solids we used the CP method we developed for molecular crystals. ${ }^{50}$

To investigate the effect of surface supercell size on the binding of benzene to the $\mathrm{Cu}$ (111) surface, we examined the TOP- $R$ benzene configuration with the vdW-DF(revPBE) functional for $(3 \times 3),(4 \times 4),(5 \times 5)$ and $(7 \times 7)$ 
surface supercells, containing 9, 16, 25 and 49 atoms per layer, respectively. We have made an online data set available ${ }^{57}$ that contains all input, output, and pseudopotential files from this work.

\section{Results and Discussion}

\section{Copper dimer, bulk copper and the performance of surface optimized basis sets}

We begin by examining the performance of our chosen functionals to reproduce the properties of a copper dimer. In Table 1 we report the bond length $(d)$, binding energy $\left(E_{B}\right)$ and vibrational frequency $(u)$. The vdW and revPBE functionals all perform reasonably well when compared to the experimental values and other traditionally high quality calculations such as B3LYP, or the "gold standard" in computational accuracy, $\operatorname{CCSD}(T)$. The vdW-DF(optB88-vdW) bond length is slightly shorter than others, and as would be expected, this leads to a slightly higher vibrational frequency. Conversely vdWDF2(rPW86) and B3LYP calculations have a slightly longer bond length, as again as expected, they produce slightly lower vibrational frequencies.

Table 1. Calculated properties of a copper dimer including bond length $(d)$, binding energy $\left(E_{B}\right)$ and vibrational frequency $(u)$. Binding energies calculated in this study include a CP correction.

\begin{tabular}{|lccc|}
\hline & $\begin{array}{c}d \\
(\AA)\end{array}$ & $\begin{array}{c}E_{B} \\
(\mathrm{eV})\end{array}$ & $\begin{array}{c}U \\
\left(\mathrm{~cm}^{-1}\right)\end{array}$ \\
\hline revPBE & 2.218 & -2.07 & 276.8 \\
vdW-DF(revPBE) & 2.237 & -2.06 & 274.4 \\
vdW-DF(optB88-vdW) & 2.201 & -2.25 & 290.7 \\
vdW-DF2(rPW86) & 2.253 & -2.21 & 256.9 \\
CCSD $(T)^{58}$ & 2.235 & -1.94 & 261.3 \\
B3LYP $^{59}$ & 2.272 & -1.85 & 243.1 \\
Experiment $^{60}$ & 2.220 & -2.01 & 266.0 \\
\hline
\end{tabular}

To examine the performance of the functionals for reproducing the properties of bulk fcc copper, we calculated the lattice parameter (a), cohesive energy $\left(E_{C}\right)$ and bulk modulus $(B)$ (see Table 2). Compared to standard exchange- correlation functionals, such as $\mathrm{PBE},{ }^{51}$ the vdWDF functionals lead to larger lattice parameters, and correspondingly smaller bulk moduli. In particular, vdW-DF(revPBE) and vdW-DF2(rPW86) have larger lattice parameters than vdW-DF(optB88-vdW). We also include in Table 2 the results from an investigation by Klimeš et $a l^{52}$ of vdW-DF functionals applied to solids. The lattice parameters from our local orbital method are all slightly larger than the corresponding planewave result of Klimeš et $a l^{52}$ They concluded that both vdW-DF(revPBE) and vdW-DF2(rPW86) functionals overestimate lattice parameters, and that vdW-DF(optB88$\mathrm{vdW}$ ) gives much better results because of the choice of the exchange enhancement factor (see Klimeš et al. $^{52}$ for more details). Our results are fully consistent with these observations.

The different exchange-correlation functionals produce a range of cohesive energies compared to the experimental value. In particular both vdW-DF(revPBE) and vdW-DF2(rPW86) functionals underestimate the cohesive energies by around $0.8 \mathrm{eV}$. The cohesive energy from the vdW-DF(optB88-vdW) functional is much better and is within about $0.1 \mathrm{eV}$ of the experimental value. The CP-corrected cohesive energies using our local orbital approach match closely (within about $0.1 \mathrm{eV}$ ) the cohesive energies calculated using the same functional but with a planewave basis set. ${ }^{52}$

Table 2. Calculated properties of bulk fcc copper, including lattice parameter (a), cohesive energy $\left(E_{C}\right)$ and bulk modulus $(B)$. Cohesive energies calculated in this study include a CP correction.

\begin{tabular}{|lccc|}
\hline & $\begin{array}{c}a \\
(\AA)\end{array}$ & $\begin{array}{c}E_{C} \\
(\mathrm{eV})\end{array}$ & $\begin{array}{c}B \\
(\mathrm{GPa})\end{array}$ \\
\hline revPBE & 3.70 & -2.96 & 118 \\
vdW-DF(revPBE) & 3.75 & -2.78 & 115 \\
vdW-DF(optB88-vdW) & 3.68 & -3.36 & 137 \\
vdW-DF2(rPW86) $^{51}$ & 3.80 & -2.69 & 105 \\
PBE $^{51}$ & 3.67 & - & 128 \\
vdW-DF(revPBE) $^{52}$ & 3.71 & -2.93 & 111 \\
vdW-DF(optB88-vdW) $^{52}$ & 3.63 & -3.52 & 138 \\
vdW-DF2(rPW86) $^{52}$ & 3.76 & -2.81 & 97 \\
Experiment $^{61}$ & 3.61 & -3.49 & 137 \\
\hline
\end{tabular}


Using the optimised bulk fcc cell for each functional, we generated (111) surface slabs containing 7 atomic layers. Following the work of Bilic et al., ${ }^{30}$ the 7-layer slab (and binding benzene to both sides of the surface slab) removes the need for dipole corrections. Garcia-Gil et al. ${ }^{51}$ investigated a number of surface optimised basis sets in their PBE study of noble metal surfaces, and showed much improved surface energies and work function values can be obtained compared to standard bulk basis sets. This motivated us to investigate how surface optimized basis sets perform with vdW-DF functionals, in order to get the best description of the $\mathrm{Cu}(111)$ surface.

In Table 3 we report the surface energy $(\sigma)$, work function (Ф) and percentage relaxation $\left(\Delta d_{12}\right)$ for the three surface optimized basis sets (as described in the computational details section) and the bulk basis set. From Table 3 we can see that in each case the three surface optimised basis sets (optimized, 1-float and 2float) display similar results to each other for both the surface energy and work function values, but vary from the bulk basis set value, particularly with reference to the work function. This general trend is observed for all the vdWDF functionals examined and the revPBE functional.

The surface optimized basis sets are arranged in the order of increasing 'quality', in reference to how well the basis set can describe the density out into the vacuum region above the surface. Firstly, the optimized basis set uses a much larger $4 s$ orbital radius (12 Bohr) to extend out the orbital out into the vacuum region. Next, the 1-float basis uses unoccupied (ghost) orbitals above the surface, located where the next atomic layer of $\mathrm{Cu}$ atoms would be in the fcc stacking sequence. Finally, the 2-float basis set should give the best description, with 2 layers of ghost orbitals above the surface (located where the next two atomic layers would be in the fcc stacking sequence). This essentially gives us a convergence trend for the surface energy and work function values, which we illustrate in Figure 1.

Table 3. Calculated surface energy $(\sigma)$, work function $(\Phi)$ and percentage relaxation $\left(\Delta d_{12}\right)$ of the copper (111) surface using the bulk and surface optimised basis sets. Calculations were performed allowing only atoms in the top and bottom layers of the slab to relax. A negative value of $\Delta d_{12}$ indicates an inward relaxation.

\begin{tabular}{|c|c|c|c|c|}
\hline & bulk & optimised & 1-float & 2-float \\
\hline \multicolumn{5}{|l|}{ revPBE } \\
\hline$\sigma(\mathrm{eV} /$ atom $)$ & 0.52 & 0.40 & 0.39 & 0.39 \\
\hline$\Phi(\mathrm{eV})$ & 3.18 & 4.41 & 4.40 & 4.47 \\
\hline$\Delta d_{12}(\%)$ & +0.22 & +0.23 & +0.23 & +0.26 \\
\hline \multicolumn{5}{|c|}{ vdW-DF(revPBE) } \\
\hline$\sigma(\mathrm{eV} /$ atom $)$ & 0.56 & 0.40 & 0.41 & 0.40 \\
\hline$\Phi(\mathrm{eV})$ & 3.21 & 4.54 & 4.55 & 4.63 \\
\hline$\Delta d_{12}(\%)$ & -1.35 & -1.33 & -1.38 & -1.36 \\
\hline \multicolumn{5}{|c|}{ vdW-DF(optB88-vdW) } \\
\hline$\sigma(\mathrm{eV} /$ atom $)$ & 0.65 & 0.51 & 0.51 & 0.51 \\
\hline$\Phi(\mathrm{eV})$ & 3.48 & 4.79 & 4.79 & 4.87 \\
\hline$\Delta d_{12}(\%)$ & -0.03 & -0.03 & -0.06 & -0.05 \\
\hline \multicolumn{5}{|c|}{ vdW-DF2(rPW86) } \\
\hline$\sigma(\mathrm{eV} /$ atom $)$ & 0.51 & 0.34 & 0.35 & 0.35 \\
\hline$\Phi(\mathrm{eV})$ & 3.41 & 4.76 & 4.77 & 4.84 \\
\hline$\Delta d_{12}(\%)$ & -0.58 & -0.58 & -0.58 & -0.53 \\
\hline
\end{tabular}

The surface optimized basis sets are arranged in the order of increasing 'quality', in reference to how well the basis set can describe the density out into the vacuum region above the surface. Firstly, the optimized basis set uses a much larger $4 s$ orbital radius (12 Bohr) to extend out the orbital out into the vacuum region. Next, the 1-float basis uses unoccupied (ghost) orbitals above the surface, located where the next atomic layer of $\mathrm{Cu}$ atoms would be in the fcc stacking sequence. Finally, the 2-float basis set should give the best description, with 2 layers of ghost orbitals above the surface (located where the next two atomic layers would be in the fcc stacking sequence). This essentially gives us a convergence trend for the surface energy and work function values, which we illustrate in Figure 1.

The surface energies for each functional in Figure 1(a) follow the same trend, with the surface energy decreasing (by around 0.1-0.2 $\mathrm{eV} /$ atom) as the basis set quality increases. This 
same trend was observed by Garcia-Gil et al. ${ }^{51}$ in their earlier PBE study using surface optimized basis sets, which we also illustrate in Figure 1(a). The vdW-DF(revPBE) and revPBE functionals converge to a surface energy of $\sim 0.4$ $\mathrm{eV} /$ atom, while the vdW-DF(optB88-vdW) and vdW-DF2(rPW86) functionals converge to surface energies of $\sim 0.5$ and $\sim 0.35 \mathrm{eV} /$ atom, respectively. The surface energy from PBE calculations with surface optimized basis sets is $\sim 0.5 \mathrm{eV} /$ atom. $^{51}$ All the calculated surface energies are less than the experimental value of $0.62 \mathrm{eV} /$ atom $\left(1.77 \mathrm{Jm}^{-2}\right){ }^{62}$ It should be noted that this experimental value is from liquid surface tension measurements and represents an "average" copper face, not specifically the (111) face. Theoretical calculations ${ }^{63}$ of the lowindex copper (111), (100) and (110) surfaces demonstrate that the surface energies are indeed different for each face.
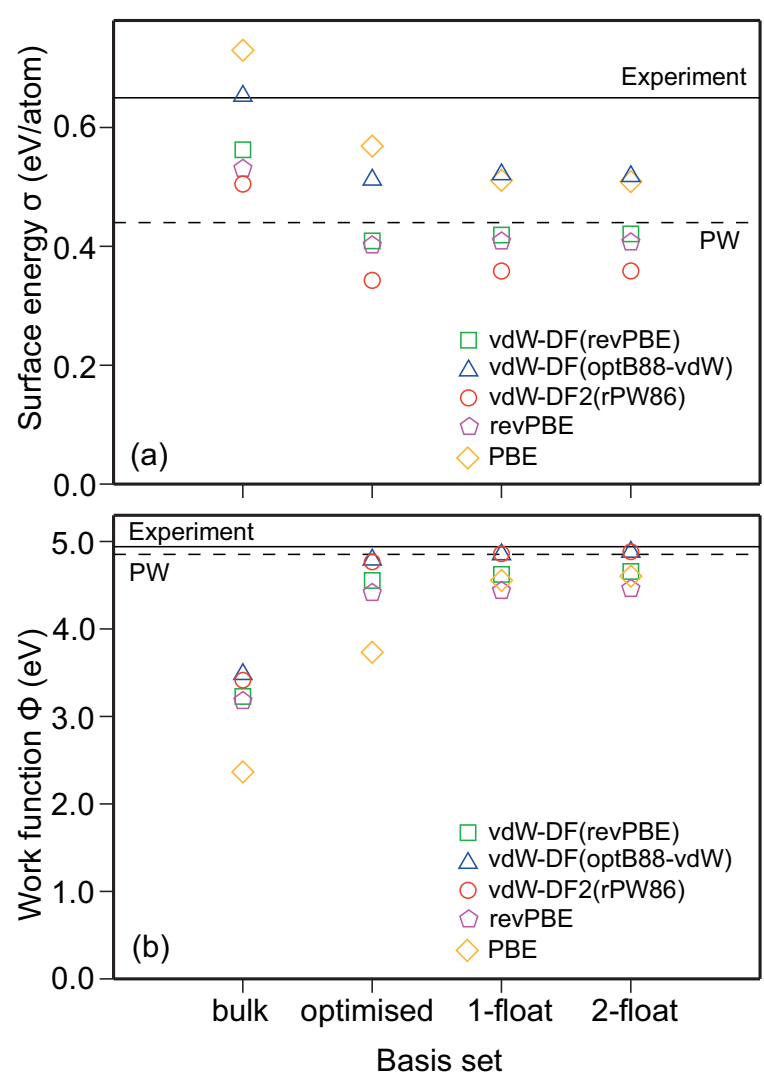

Figure 1. Plot of the calculated (a) surface energy and (b) work function for each basis set option for a clean copper (111) surface. The PBE results are taken from Garcia-Gil et al. ${ }^{51}$ as well as their planewave (PW) results, also using the PBE functional. The experimental values of the surface energy ${ }^{64}$ and work function ${ }^{62}$ are also shown.

Figure 1(b) shows that the work function calculated with each functional increases as the basis set quality increases. The work function value using the bulk basis set with all functionals is underestimated by up to several $\mathrm{eV}$ compared to the experimental value, but is substantially improved by the use of the surface optimized basis sets. The revPBE, vdWDF(revPBE), vdW-DF(optB88-vdW) and vdWDF2(rPW86) functionals converge to work function values of approximately 4.45, 4.6, 4.8 and $4.8 \mathrm{eV}$, respectively. The vdW-DF results in particular converge to values close to the work function of $\sim 4.85 \mathrm{eV}$ from PBE planewave calculations, $^{51}$ and the experimental value of $4.94 \mathrm{eV}^{64}$. Earlier PBE calculations ${ }^{51}$ follow a similar trend.

The percentage relaxations $\left(\Delta d_{12}\right)$ in Table 3 show that for a particular functional, the choice of basis set (bulk or surface optimised) has little effect on the relaxation of the outermost layers of the surface slab. The percentage relaxations, whilst quite small, do vary with the choice of functional, with revPBE having a small outward relaxation of approximately $0.24 \%$, and vdWDF(revPBE), vdW-DF(optB88-vdW) and vdWDF2(rPW86) having inward relaxations of -1.36 , -0.04 and $-0.57 \%$, respectively. Theoretical investigations using the PBE functional report inward relaxations of $-1.19 \%{ }^{65}$ or $-0.56 \%$, ${ }^{66}$ while calculations using the local density approximation (LDA) calculations report a value of $-1.56 \%{ }^{65}$ Experimentally, low-energy electron diffraction studies report relaxations of $-0.3 \pm 1.0 \%{ }^{67}$ and $-0.7 \pm 0.5 \% .{ }^{68}$

Overall, we find good agreement between our results and other theoretical and experimental studies for determining the properties of the 
copper (111) surface. Based on the basis set convergence results for the work function and surface energy values, the 1-float basis appears to be the best compromise between converged surface properties and computational efficiency.

\section{Effect of the surface supercell size on geometries and binding energies}

Carrasco et al. $^{32}$ remark in their recent investigation of benzene interactions on transition metal surfaces that using a 9-atoms per layer $(3 \times 3)$ supercell ensures negligible interaction between periodic images of the benzene molecule. However, in an earlier computational study of benzene on the copper (111) surface using the same $(3 \times 3)$ surface slab, Berland et al. ${ }^{29}$ report that their calculations contain an error due to adsorbate-adsorbate interactions. They report that it is difficult to quantify, but approximate a value of $\sim 6 \mathrm{meV}$, which is substantially smaller ( $\sim 1 \%$ of the value) than their average binding energy for benzene configurations of $-0.54 \mathrm{eV}$. A recent investigation of benzene on transition metal surfaces by Yildirim et al. ${ }^{39}$ uses a 16-atoms per layer $(4 \times 4)$ surface slab.

To justify using a $(5 \times 5)$ surface slab and to attempt to quantify the errors due to periodic overlap of benzene molecules in the $(3 \times 3)$ supercell, we performed calculations of the TOP- $R$ benzene configuration using the vdWDF(revPBE) functional in $(3 \times 3),(4 \times 4),(5 \times 5)$ and (7x7) surface slabs (containing 9, 16, 25 and 49 atoms per layer, respectively).

We begin by first examining the optimized geometries of the $(3 \times 3)$ surface slab calculation. The separation distance between a benzene molecule and the $\mathrm{Cu}$ (111) surface with the (3x3) surface slab calculation is $3.46 \AA$, whereas the distance is $3.40 \AA$ in the $(4 \times 4)$ surface slab and $3.39 \AA$ for the $(5 \times 5)$ and $(7 \times 7)$ surface slabs. So the first consequence of using only a $(3 \times 3)$ surface slab is that we introduce an error of $0.07 \AA$ in the separation distance - which will also have an impact on the calculated binding energy. The separation distances in both the

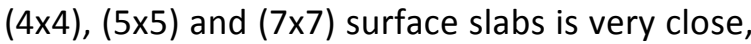
with only a $0.01 \AA$ difference from the $(4 \times 4)$ surface slab, suggesting good convergence at this value.

Examining the optimized geometry of the benzene molecule in the $(3 \times 3)$ surface slab, the closest distance between periodic repeats of a benzene molecule is a $\mathrm{H}$...H distance of $2.98 \AA$ (the smallest C...C distance is $5.15 \AA$ ). Our basis set uses 7 Bohr cutoffs for the $s$ orbitals of $\mathrm{H}$ and $\mathrm{C}$ atoms, so the minimum distance without overlap between any of the $\mathrm{C}$ or $\mathrm{H}$ atoms is $~ 7.5$ $\AA$. Clearly, not only do the orbital tails of the $\mathrm{H}$ atoms overlap between periodic images, but also the orbital tails of the $C$ atoms as well. In the $(4 \times 4)$ surface slab, the closest distance between periodic repeats of benzene molecules is a $\mathrm{H} . . . \mathrm{H}$ distance of $5.62 \AA$, also much shorter than the minimum $\sim 7.5 \AA$ separation that is required. The smallest C...C distance is $7.79 \AA$, just larger than the minimum required separation. This suggests that both the $(3 \times 3)$ and $(4 \times 4)$ surface slab will have an interaction error from the periodic repeats, although to a lesser extent in the case of the $(4 \times 4)$ cell. In the $(5 \times 5)$ and $(7 \times 7)$ surface slabs, the closest distances between periodic repeats are $\mathrm{H} . . . \mathrm{H}$ distances of 8.27 and $13.56 \AA$, respectively (the corresponding smallest $C$...C distances are 10.45 and $15.76 \AA$, respectively). This suggests that the $(5 \times 5)$ and $(7 \times 7)$ surface slabs are large enough to avoid overlap of the $\mathrm{C}$ and $\mathrm{H}$ orbitals from neighboring periodic repeats of the benzene molecules.

Examining how the binding energy is affected by the choice of surface slab size is a complicated problem, because we need to separate the different contributions to the energy. Simply comparing the counterpoise corrected (CP) binding energies will not give the best assessment because the CP correction will not only give a correction for BSSE arising from overlap between the benzene molecule and the copper surface, but will also have a contribution from a benzene molecule interacting with its 
periodic images. Instead, we focus on the energies of the benzene molecules in their optimized TOP- $R$ orientations. For the $(5 \times 5)$ and (7x7) surface slabs, the energy of the benzene molecules are essentially identical $(<1 \mathrm{meV}$ difference), but for the $(3 \times 3)$ and $(4 \times 4)$ surface slabs, the energy is lowered (more negative) by 73 and $3 \mathrm{meV}$, respectively. Other than a possible contribution from the distortion of the molecule itself, this energy lowering of the benzene molecule must be caused by it interacting with periodic images of itself. To assess the possibility that distortion of the benzene geometry is causing the energy lowering, we took the optimized benzene molecule geometry from the $(3 \times 3)$ surface slab and placed it in the $(5 \times 5)$ surface slab to do a single point calculation. The resulting energy was less than $1 \mathrm{meV}$ different from the energy when we optimized the benzene molecule in the $(5 \times 5)$ surface slab. This confirms that it is not distortion of the benzene molecule causing the energy differences, leaving the interaction with periodic images as the only other possible cause. So using a $(3 \times 3)$ surface slab, rather than a $(5 \times 5)$ or $(7 \times 7)$ surface slab introduces an error in the energy of the benzene molecule by $\sim 70$ meV. Given the average $C P$ corrected binding energy with vdW-DF(revPBE) in this study is $-0.40 \mathrm{eV}$, this represents about a $17 \%$ overbinding caused by this problem, along with a $0.07 \AA$ error in the separation distance between the benzene molecule and the $\mathrm{Cu}(111)$ surface. The error here is substantially more than the estimated $1 \%$ error of Berland et al. ${ }^{29}$ Using a (4x4) surface slab, the errors are much reduced, with only small errors in the separation distance
(0.01 $\AA$ ) and binding energy ( 1 \%). These findings of course are for only one of the vdWDF functionals used in this study and for only one of the eight high symmetry benzene orientations, but it certainly provides a good estimate for quantifying the errors introduced into calculations by using a simulation cell that is too small.

The results above not only serve to quantify the errors introduced when using a $(3 \times 3)$ and $(4 \times 4)$ surface slab, but also justify our choice of using the $(5 \times 5)$ surface slab for docking calculations.

\section{Binding energies of the eight high-symmetry orientations of benzene}

We calculated eight high-symmetry docking orientations of benzene on the $\mathrm{Cu}$ (111) surface, denoted BR-I, FCC-I, HCP-I, TOP-I, BR-R, FCC- $R$, HCP- $R$, and TOP- $R$. We use the same notation as described by Berland et al., ${ }^{29}$ where "l" refers to in-phase orientations and " $R$ " refers to a $30^{\circ}$ rotation of the benzene molecule with respect to the "I" orientations. For our analysis of the binding energies of benzene on copper and associated properties like the separation distance and work function, we have split the results into two sections. Here we examine the binding energies of the eight individual configurations, while in next section we discuss the average binding energies and associated properties. In Table 4 we report the $\mathrm{CP}$ corrected binding energies for each configuration and compare them with the results of other computational studies.

The binding energy results in Table 4 highlight

Table 4. Calculated binding energies (eV) for the eight high-symmetry docking orientations of benzene on the copper (111) surface. Binding energies in this study are CP corrected.

\begin{tabular}{|lccccccc|}
\hline & revPBE & $\begin{array}{c}\text { vdW-DF } \\
(\text { revPBE) }\end{array}$ & $\begin{array}{c}\text { vdW-DF } \\
(\text { optB88-vdW) }\end{array}$ & $\begin{array}{c}\text { vdW-DF2 } \\
(\text { rPW86) }\end{array}$ & $\begin{array}{c}\text { vdW-DF } \\
(\text { revPBE) }\end{array}$ & PW-91 $^{30}$ & RPBE-XDM $^{33}$ \\
\hline BR-I & +0.08 & -0.41 & -0.67 & -0.38 & -0.55 & -0.02 & -0.60 \\
FCC-I & +0.03 & -0.41 & -0.64 & -0.37 & -0.55 & -0.02 & -0.61 \\
HCP-I & +0.06 & -0.39 & -0.65 & -0.35 & -0.55 & -0.03 & -0.60 \\
TOP-I & +0.07 & -0.38 & -0.64 & -0.41 & -0.53 & +0.04 & -0.60 \\
BR-R & +0.04 & -0.41 & -0.63 & -0.37 & -0.53 & -0.02 & -0.59 \\
FCC- $R$ & +0.05 & -0.43 & -0.63 & -0.38 & -0.53 & -0.02 & -0.63 \\
HCP-R & +0.08 & -0.41 & -0.65 & -0.37 & -0.53 & -0.02 & -0.62 \\
TOP-R & +0.09 & -0.40 & -0.62 & -0.37 & -0.52 & +0.01 & -0.58 \\
Average & +0.06 & -0.40 & -0.64 & -0.38 & -0.54 & -0.01 & -0.60 \\
\hline
\end{tabular}


why a good description of van der Waals forces is so important in this adsorption system. Using standard DFT functionals like revPBE or PW-91, which do not account for van der Waals forces, there is essentially no favourable interaction between benzene and the $\mathrm{Cu}(111)$ surface. The average binding energy for revPBE is $+0.06 \mathrm{eV}$, while Bilic et al. $^{30}$ reported an average binding energy of $-0.01 \mathrm{eV}$ for PW-91. Using vdW functionals there is a favourable interaction of benzene with the $\mathrm{Cu}$ (111) surface, with vdWDF(revPBE), vdW-DF(optB88-vdW) and vdWDF2(rPW86) calculations producing average binding energies of $-0.40,-0.64$ and $-0.38 \mathrm{eV}$, respectively.

The binding energies of the eight configurations for any particular functional are, not surprisingly, very similar. The vdW-DF(optB88$\mathrm{vdW}$ ) method predicts that the BR-I orientation of benzene is most stable, while vdWDF(revPBE) and RPBE-XDM methods ${ }^{33}$ calculate FCC- $R$ is most stable, and vdW-DF2(rPW86) predicts the TOP-I orientation. DFT calculations coupled with experimental vibrational analysis for benzene interacting with other metal surfaces including Pt, Pd and Rh $(111)^{69,70}$ have suggested that the bridge $B R-I$ is the more favourable orientation. Although the energy differences are small, the vdW-DF(optB88-vdW) calculations also predict this as the most preferred orientation.

The magnitude of the vdW-DF(optB88-vdW) binding energies are very similar to those from RPBE-XDM ${ }^{33}$ calculations while our vdWDF(revPBE) and vdW-DF2(rPW86) binding energies are about $0.2 \mathrm{eV}$ higher. The vdWDF(revPBE) results from Berland et al. ${ }^{29}$ fall somewhere between these two, however these differences from our vdW-DF(revPBE) values could be because they use a much smaller $(3 \times 3)$ supercell, which introduces binding energy errors as detailed earlier, and they use the PBE functional, which does not account for $\mathrm{vdW}$ interactions, to optimize geometries, and use a fixed substrate surface.
Average binding energies, separation distance and change in work function

We now compare the average binding energies $\left(E_{B}\right)$ for our vdW calculations of benzene on the $\mathrm{Cu}$ (111) surface to results from other theoretical calculations and available experimental data. We also examine the separation distance $(d)$ between the benzene molecule and the copper surface, and calculate the change in work function $(\Delta \Phi)$, using the method described by Witte et al. ${ }^{38}$ These results are reported in Table 5.

Table 5. Average binding energy $\left(E_{B}\right)$, separation distance $(d)$ and change in work function $(\Delta \Phi)$ of benzene on the $\mathrm{Cu}$ (111) surface using various computational methods, compared to available experimental data.

\begin{tabular}{|c|c|c|c|}
\hline & $\begin{array}{c}E_{B} \\
(\mathrm{eV})\end{array}$ & $\begin{array}{c}d \\
(\AA)\end{array}$ & $\begin{array}{l}\Delta \Phi \\
(\mathrm{eV})\end{array}$ \\
\hline revPBE & +0.06 & 4.00 & -0.31 \\
\hline vdW-DF(revPBE) & -0.40 & 3.38 & -0.69 \\
\hline vdW-DF(optB88-vdW) & -0.64 & 2.93 & -1.18 \\
\hline vdW-DF2(rPW86) & -0.38 & 3.32 & -0.72 \\
\hline PW91 ${ }^{30}$ & -0.01 & 3.60 & - \\
\hline $\mathrm{PBE}^{37}$ & -0.05 & 3.70 & -0.37 \\
\hline $\mathrm{MP} 2^{38}$ & - & 4.00 & -1.08 \\
\hline$M P 2^{28,31}$ & -0.35 & 3.60 & -1.08 \\
\hline$v d W-D F(r e v P B E)^{37}$ & -0.55 & 3.70 & - \\
\hline vdW-DF(revPBE) $)^{32}$ & -0.50 & 4.14 & - \\
\hline$v d W-D F(\operatorname{revPBE})^{29}$ & -0.52 & 3.75 & - \\
\hline vdW-DF(revPBE) $)^{39}$ & -0.53 & 3.44 & - \\
\hline vdW-DF2(rPW86) & -0.47 & 3.38 & - \\
\hline vdW-DF2(rPW86) & -0.49 & 3.31 & - \\
\hline vdW-DF(optB88-vdW) ${ }^{32}$ & -0.68 & 3.08 & - \\
\hline$v d W-D F(o p t B 88-v d W)^{39}$ & -0.74 & 3.08 & - \\
\hline RPBE-XDM ${ }^{33}$ & -0.60 & 3.00 & -1.18 \\
\hline Expt. & $\begin{array}{c}-0.59^{28,46} \\
-0.62^{45}\end{array}$ & - & $-1.05^{38}$ \\
\hline Expt. Deduced & - & $2.90^{37}$ & - \\
\hline
\end{tabular}

The results in Table 5 highlight that binding energies should not be evaluated in isolation, but rather there is a clear relationship between the binding energy, the change in work function and the separation distance between the benzene molecule and the copper surface. The experimental binding energy has been reported as approximately $-0.60 \mathrm{eV}^{28,45,46}$ and the change in work function as $-1.05 \mathrm{eV}^{38}$ however the 
measurement of the separation distance of the benzene molecule from the surface has to date not been achieved. Toyoda et al. ${ }^{37}$ have deduced an experimental separation distance for benzene on $\mathrm{Cu}, \mathrm{Ag}$ and $\mathrm{Au}$ (111) surfaces by extrapolating the experimental change in work function values, combined with their vdWDF(revPBE) DFT calculations. In the absence of actual experimental measurements, we use their extrapolated value of $2.90 \AA$ as a guide for assessing the separation distances with the various computational methods.

When using the revPBE, $\mathrm{PW} 911^{30}$ or $\mathrm{PBE}^{37}$ functionals that do not include vdW forces, there is negligible or no interaction between benzene and the $\mathrm{Cu}(111)$ surface. The change in work function values are much less than the experimental value, with revPBE and PBE values of -0.31 and $-0.37 \mathrm{eV}$, respectively. Correspondingly, the separation distances from these methods are all much larger (distances range from $3.60-4.00 \AA$ ) than the experimentally deduced separation of $2.9 \AA$.

Wave function methods such as MP2 do account for dispersion forces and report accurate values for the change in work function, however the separation distances are again large, ranging from $3.60-4.00 \AA^{28,31,38}$ much larger than the experimentally deduced value, and the subsequent binding energy of $-0.35 \mathrm{eV}$ is underestimated compared to the experimental value of around $-0.60 \mathrm{eV}$. In these studies, the copper surface is fixed at the bulk positions, and only the benzene molecule is relaxed, which could account for some of the error in the separation distance and subsequent binding energy.

The vdW-DF(revPBE) functional of Dion et al. ${ }^{21}$ noticeably improves the results over functionals that don't include vdW interactions. While the separation distance of $3.38 \AA$ is an improvement, it is still about $0.5 \AA$ larger than the experimentally deduced value. The longer separation distance leads to under-binding, with a binding energy of $-0.40 \mathrm{eV}$, which is around $-0.2 \mathrm{eV}$ less than the experimental value. The change in work function is $-0.69 \mathrm{eV}$, which is also underestimated by around $0.4 \mathrm{eV}$. While the binding energies from other calculations using the vdW-DF(revPBE) functional are slightly larger at around $-0.5 \mathrm{eV}$, there is a substantial spread of separation distances, which range from 3.44 to 4.14 $\AA . .^{29,32,37,39}$ The majority of these studies use only a $(3 \times 3)$ supercell, which as we reported in eariler, leads to interactions between periodic images of the benzene molecule, resulting in small changes in the separation distances and over-estimating the binding energy. Two of these studies ${ }^{29,37}$ optimize the geometries using the PBE functional, which does not account for $v d W$ interactions, leading to larger separation distances ranging from 3.70-3.75 A. Planewave vdW-DF(revPBE) calculations by Yildirim et al. ${ }^{39}$ report a separation distance of $3.44 \AA$. This value is close to our value of $3.38 \AA$, which is the average separation distance of the eight highsymmetry configurations we investigated. In contrast, Yildirim et al. ${ }^{39}$ only examined the $\mathrm{Br}-\mathrm{I}$ configuration, which may account for these small differences. The separation distance of $4.14 \AA ̊$ reported by Carrasco et al. ${ }^{32}$ is somewhat surprising as this distance is substantially larger than Yildirim et al. and our value of around 3.4 $\AA$, and is even larger than the separation distances calculated by functionals that don't include vdW interactions (e.g. PBE, PW91 and revPBE).

The results from the vdW-DF2(rPW86) functional are not significantly different to those from the vdW-DF(revPBE) results. The binding energy of $-0.38 \mathrm{eV}$ is almost identical to the vdW-DF(revPBE) value of $-0.40 \mathrm{eV}$, while the separation distance and change in work function values of $3.32 \AA$ and -0.72 , respectively, are slightly improved over vdWDF(revPBE) results. Other calculations ${ }^{32,39}$ using vdW-DF2(rPW86) report slightly larger binding energies $(-0.47$ and $-0.49 \mathrm{eV})$ but no change in work function values. Interestingly, Yildirim et al. ${ }^{39}$ report a separation distance of $3.31 \AA$, almost identical to our $3.32 \AA$, whereas 
Carrasco et $a .^{32}$ report a distance of $3.38 \AA$. Yildirim et al. use a $(4 \times 4)$ supercell, while Carrasco et al. use a $(3 \times 3)$ supercell for calculations. This larger separation distance is likely a result of using a too small simulation cell. For molecular crystals, we have found ${ }^{50}$ that vdW-DF2(rPW86) produces superior geometries and lattice energies to $\mathrm{vdW}$ DF(revPBE), but here both perform similarly. This can be largely attributed to the strong repulsive character of both functionals at close range, leading to a reduction in binding energies, $^{39}$ while at long distance they have similar tails, leading to the similar behaviour.

The use of the optimised vdW-DF(optB88-vdW) functional leads to significantly improved results in almost all aspects. The separation distance of $2.93 \AA$ is very close to the experimentally deduced value. The corresponding binding energy and change in work function values of $-0.64 \mathrm{eV}$ and $-1.18 \mathrm{eV}$ are also close to the experimental values of around -0.60 and $-1.05 \mathrm{eV}$, respectively. Similar vdW-DF(optB88-vdW) calculations report a slightly larger separation distance of $3.08 \AA$, and binding energies of $0.68^{32}$ and $0.74^{39} \mathrm{eV}$. As discussed earlier, Carrasco et $a .^{32}$ use a small (3x3) surface cell, which we found for vdWDF(revPBE) leads to a $17 \%$ overbinding error in the binding energy and a larger separation distance (by $0.07 \AA$ ). Although we didn't do our supercell convergence testing with the vdWDF(optB88-vdW) functional, given the basis set is the same, it is not unreasonable to assume similar errors exist with this functional too in the smaller $(3 \times 3)$ cell. Both of these studies also use only the $\mathrm{Br}-\mathrm{I}$ orientation for benzene molecules, whereas we examine all eight highsymmetry configurations, which will lead to some small variation in values. The optimised vdW-DF functionals were introduced to address the problem of large separation distances with the $v d W-D F(r e v P B E)$ and $v d W-D F 2(r P W 86)$ functionals, and use less repulsive exchange functionals to produce improved accuracy in many systems. ${ }^{23,39,52,71}$ The RPBE-XDM functional also produces similarly excellent results, $^{33}$ with a binding energy of $-0.60 \mathrm{eV}$, separation distance of $3.00 \AA$ and change in work function of $-1.18 \mathrm{eV}$. The authors also use a small ( $3 \times 3)$ supercell calculation, so we expect there will be some over-binding error in the binding energies and a small separation distance error.

\section{Conclusions}

We have investigated the performance of the several vdW functionals using the local orbital SIESTA code to examine the interactions of benzene on the copper (111) surface. In particular we use the vdW-DF(revPBE), vdWDF(optB88-vdW), vdW-DF2(rPW86) functionals and compare the results to the revPBE functional, which does not account for vdW interactions. For calculations of the pure $\mathrm{Cu}$ (111) surface we examined the surface energies, work functions and layer relaxations, and demonstrated surface optimized basis sets produce superior results to standard bulk basis sets for vdW functionals. We investigated the effect of supercell size on the separation distance and binding energy using vdW$D F(\operatorname{revPBE})$, and demonstrated the routinely used $(3 \times 3)$ supercell has an overbinding error of $17 \%$ in the binding energy, and the separation distance is $0.07 \AA$ larger as the result of spurious interactions between periodic images of the benzene molecule. We also showed these errors are significantly reduced for a $(4 \times 4)$ supercell and essentially non-existent for a (5x5) supercell, and consequently used a $(5 \times 5)$ supercell for all docking calculations. We examined the binding of the 8 high-symmetry orientations of benzene on the $\mathrm{Cu}$ (111) surface, and calculated the binding energy, separation distance and change in work function. The revPBE functional essentially shows no favourable interaction between benzene and the copper surface as expected. The vdW-DF(revPBE) and vdW-DF2(rPW86) functionals produce similar results, underestimating the binding energies and change in work function, while over-estimating the separation distances. The vdW-DF(optB88- 
$v d W)$ functional produces the most promising results, and closely matches the experimental and experimentally deduced values. Our results with all functionals studied are largely consistent with a number of other studies using these functionals. The optimised vdW-DF functional vdW-DF(optB88-vdW) was one of several introduced to address the problems of large separation distances with the vdWDF(revPBE) and vdW-DF2(rPW86) functionals, and here provide superior results for studying benzene on copper surfaces.

Finally we have clearly demonstrated that a local orbital approach performs equally as well as planewave approaches. This is advantageous as it allows for the use of larger vacuum gaps at essentially no additional computational cost as well as larger simulation cells, both crucial when examining adsorption of larger molecules on surfaces.

\section{Acknowledgments}

We gratefully acknowledge the $\mathrm{NCl}$ and iVEC for the provision of computational resources. The authors also thank M.J. Ford for useful discussions.

Keywords: vdW-DF, vdW-DF2, van der Waals, copper surfaces, benzene

\section{References and Notes}

1. S. Grimme. WIREs Comput. Mol. Sci. 2011, 1, 211-228.

2. B. Civalleri, C. M. Zicovich-Wilson, L. Valenzano, P. Ugliengo. CrystEngComm 2008, 10, 405-410.

3. S. Grimme. J. Comput. Chem. 2004, 25, 1463-1473.
4. M. A. Neumann, F. J. J. Leusen, J. Kendrick. Angew. Chem., Int. Ed. 2008, 47, 2427-2430.

5. K. E. Riley, J. Vondrasek, P. Hobza. Phys. Chem. Chem. Phys. 2007, 9, 5555-5560.

6. R. W. Williams, D. Malhotra. Chem. Phys. 2006, 327, 54-62.

7. A. Tkatchenko, M. Scheffler. Phys. Rev. Lett. 2009, 102, 073005.

8. T. Sato, H. Nakai. J. Chem. Phys. 2009, 131, 224104.

9. S. Grimme, J. Antony, S. Ehrlich, H. Krieg. J. Chem. Phys. 2010, 132, 154104.

10. A. D. Becke, E. R. Johnson. J. Chem. Phys. 2005, 122, 154104.

11. A. D. Becke, E. R. Johnson. J. Chem. Phys. 2006, 124, 014104.

12. A. Hebelmann. J. Chem. Phys. 2009, 130, 084104.

13. A. Otero-de-la-Roza, E. R. Johnson. J. Chem. Phys. 2012, 136, 174109.

14. J.-D. Chai, M. Head-Gordon. Phys. Chem. Chem. Phys. 2008, 10, 6615-6620.

15. X. Xu, W. A. Goddard III. Proc. Natl. Acad. Sci. USA 2004, 101, 2673-2677.

16. Y. Zhao, D. G. Truhlar. J. Phys. Chem. A 2005, 109, 5656-5667.

17. Y. Zhao, D. G. Truhlar. Acc. Chem. Res. 2008, 41, 157-167.

18. T. Schwabe, S. Grimme. Phys. Chem. Chem. Phys. 2007, 9, 3397-3406.

19. Y. Zhang, X. Xu, W. A. Goddard III. Proc. Natl. Acad. Sci. USA 2009, 106, 4963-4968.

20. V. Cooper. Physical Review B 2010, 81, 161104.

21. M. Dion, H. Rydberg, E. Schroder, D. C. Langreth, B. I. Lundqvist. Phys. Rev. Lett. 2004, 92, 246401.

22. K. Lee, E. D. Murray, L. Kong, B. I. Lundqvist, D. C. Langreth. Phys. Rev. B 2010, $82,081101$. 
23. J. Klimeš, D. R. Bowler, A. Michaelides. J. Phys.: Condens. Matter 2010, 22, 022201.

24. O. A. Vydrov, T. Van Voorhis. Phys. Rev. Lett. 2009, 103, 063004.

25. B. de Boer, A. Hadipour, M. M. Mandoc, T. van Woudenbergh, P. W. M. Blom. Adv. Mater. 2005, 17, 621-625.

26. X. Guo, M. Myers, S. Xiao, M. Lefenfeld, R. Steiner, G. S. Tulevski, J. Tang, J. Baumert, F. Leibfarth, J. T. Yardley, M. L. Steigerwald, P. Kim, C. Nuckolls. Proc. Natl. Acad. Sci. USA 2006, 103(31), 11452-11456.

27. J. Genzer, K. Efimenko. Science 2000, 290, 2130-2133.

28. P. S. Bagus, K. Hermann, C. Woll. J. Chem. Phys. 2005, 123, 184109.

29. K. Berland, T. L. Einstein, P. Hyldgaard. Phys. Rev. B 2009, 80, 155431.

30. A. Bilic, J. R. Reimers, N. S. Hush, R. C. Hoft, M. J. Ford. J. Chem. Theory Comput. 2006, 2(4), 1093-1105.

31. R. Caputo, B. P. Prascher, V. Staemmler, P. S. Bagus, C. Woll. J. Phys. Chem. A 2007, 111, 12778-12784.

32. J. Carrasco, W. Liu, A. Michaelides, T. Tkatchenko. J. Chem. Phys. 2014, 140, 084704.

33. T. S. Chwee, M. B. Sullivan. J. Chem. Phys. 2012, 137, 134703.

34. H. Lesnard, M.-L. Bocquet, N. Lorente. J. Am. Chem. Soc. 2007, 129(14), 4298-4305.

35. H. Lesnard, N. Lorente, M.-L. Bocquet. J. Phys.: Condens. Matter 2008, 20, 224012.

36. K. Tonigold, A. Gross. J. Chem. Phys. 2010, 132, 224701.

37. K. Toyoda, Y. Nakano, I. Hamada, K. Lee, S. Yanagisawa, Y. Morikawa. Surf. Sci. 2009, 603, 2912-2922.

38. G. Witte, S. Lukas, P. S. Bagus, C. Woll. Appl. Phys. Lett. 2005, 87, 263502.

39. H. Yildirim, T. Greber, A. Kara. J. Phys.

Chem. C 2013, 117, 20572-20583.
40. L. A. Zotti, G. Teobaldi, W. Palotas, W. Ji, H.-J. Gao, W. A. FHofer. J. Comput. Chem. 2008, 29, 1589-1595.

41. L. Zoppi, A. Garcia, K. K. Baldridge. J. Phys. Chem. A 2010, 114, 8864-8872.

42. M. Vanin, J. J. Mortensen, A. K. Kelkkanen, J. M. Garcia-Lastra, K. S. Thygesen, K. W. Jacobsen. Phys. Rev. B 2010, 81, 081408.

43. L. Romaner, D. Nabok, P. Puschnig, E. Zojer, C. Ambrosch-Draxl. New J. Phys. 2009, 11, 053010.

44. S. J. Jenkins. Proc. R. Soc. A 2009, 465, 2949-2976.

45. M. Xi, M. X. Yang, S. K. Jo, B. E. Bent, P. Stevens. J. Chem. Phys. 1994, 101, 91229131.

46. S. Lukas, S. Vollmer, G. Witte, C. Woll. J. Chem. Phys. 2001, 114, 10123-10130.

47. S. D. Kevan, R. H. Gaylord. Phys. Rev. B 1987, 36, 5809-5818.

48. J. M. Soler, E. Artacho, J. D. Gale, A. Garcia, J. Junquera, P. Ordejon, D. SanchezPortal. J. Phys.: Condens. Matter 2002, 14, 2745-2779.

49. D. J. Carter, A. L. Rohl. J. Chem. Theory Comput. 2012, 8, 281-289.

50. D. J. Carter, A. L. Rohl. J. Chem. Theory Comput. 2014, DOI: 10.1021/ct500335b.

51. S. Garcia-Gil, A. Garcia, N. Lorente, P. Ordejon. Phys. Rev. B 2009, 79(7), 075441.

52. J. Klimeš, D. R. Bowler, A. Michaelides. Phys. Rev. B 2011, 83, 195131.

53. Y. Zhang, W. Yang. Phys. Rev. Lett. 1998, $80,890$.

54. N. Troullier, J. L. Martins. Phys. Rev. B 1991, 43, 1993-2006.

55. J. Junquera, O. Paz, D. Sanchez-Portal, E. Artacho. Phys. Rev. B 2001, 64, 235111. 56. S. F. Boys, F. Bernardi. Mol. Phys. 1970, 19, 553-566. 
57. D. J. Carter, A. L. Rohl, Input and output files from vdW calculations of benzene on the copper (111) surface: Available at http://doi.org/10.4225/06/4ED6B979EBHE7 , accessed on July 15, 2014.

58. D. Danovich, M. Filatov. J. Phys. Chem. A 2008, 112(50), 12995-13001.

59. X. Wang, X. Wan, H. Zhou, S. Takami, M. Kubo, A. Miyamoto. J. Mol. Struct: Theochem 2002, 579(1-3), 221-227.

60. M. D. Morse. Chem. Rev. 1986, 86, 1049-1109.

61. C. Kittel. Introduction to Solid State Physics; John Wiley \& Sons: New York, 1996.

62. W. R. Tyson, W. A. Miller. Surf. Sci. 1977, 62, 267-276.

63. M. J. Mehl, D. A. Papaconstantopoulos. Phys. Rev. B 1996, 54, 4519-4530.

64. F. R. De Boer, R. Boom, W. C. M. Mattens, A. R. Miedema, A. K. Niessen.
Cohesion in Metals; North-Holland: Amsterdam, 1988.

65. J. L. F. Da Silva, C. Stampfl, M. Scheffler. Surf. Sci. 2006, 600, 703-715.

66. J. L. F. Da Silva, K. Schroeder, S. Blugel. Phys. Rev. B 2004, 69, 245411.

67. S. P. Tear, K. Roll, M. Prutton. J. Phys. C: Solid State Phys. 1981, 14, 3297-3311.

68. S. A. Lindgren, L. Wallden, J. Rundgren, P. Westrin. Phys. Rev. B 1984, 29, 576-588.

69. C. Morin, D. Simon, P. Sautet. J. Phys. Chem. B 2004, 108, 5653-5665.

70. M. Saeys, M.-F. Reyniers, G. B. Marin. J. Phys. Chem. B 2002, 106, 7489-7498.

71. F. Mittendorfer, A. Garhofer, J. Redinger, J. Klimeš, J. Harl, G. Kresse. Phys. Rev. B 2011, 84, 201401. 


\section{GRAPHICAL ABSTRACT}

Damien J. Carter and Andrew L. Rohl

van der Waals corrected density functional calculations of the adsorption of benzene on the $\mathrm{Cu}(111)$ surface

Here we investigate the performance of several van der Waals (vdW) functionals at calculating the interactions between benzene and the copper (111) surface. We demonstrate that local orbital methods using appropriate basis sets combined with a vdW functional can successfully model adsorption between metal surfaces and organic molecules.

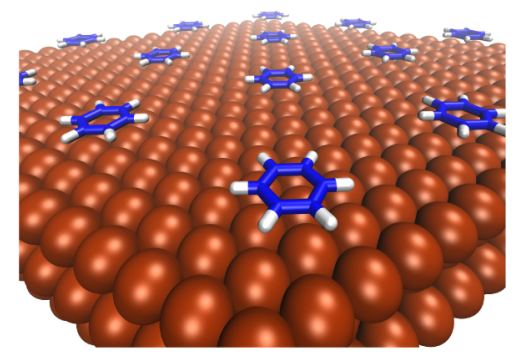

